\title{
A Linearly Implicit Finite-Difference Scheme for the One-Dimensional Porous Medium Equation
}

\author{
By David Hoff
}

\begin{abstract}
We present and analyze a linearly implicit finite-difference scheme for computing approximate solutions and interface curves for the porous medium equation in one space variable. Our scheme requires only that linear, tridiagonal systems of equations be solved at each time step. We derive error bounds for the approximate interface curves as well as for the approximate solutions under the rather mild mesh condition $\Delta t / \Delta x \leqslant$ constant.
\end{abstract}

1. Introduction. In this paper we present and analyze a linearly implicit finitedifference scheme for computing approximate solutions and interface curves for the porous medium equation in one space variable:

$$
\begin{gathered}
\frac{\partial v}{\partial t}=m v \frac{\partial^{2} v}{\partial x^{2}}+\frac{m}{m-1}\left(\frac{\partial v}{\partial x}\right)^{2}, \quad v=v(x, t), t>0, x \in \mathbf{R} ; \\
v(x, 0)=v_{0}(x) .
\end{gathered}
$$

Here $v$ denotes the pressure in a polytropic fluid occupying a portion of $x$-space, $t$ is time, and the constant $m>1$ is the adiabatic constant of the fluid. Equation (1.1) results by coupling D'Arcy's law with the Euler equation for conservation of mass; see [10]. It is known (see below) that if $v_{0}$ has compact support, then so does $v(\cdot, t)$. The "interface curves" are therefore defined by spt $v(\cdot, t)=\left[\zeta_{l}(t), \zeta_{r}(t)\right]$.

Our difference scheme requires only that linear, tridiagonal systems of equations be solved at each time step. We derive error bounds, not only for the approximate solutions, but also for the approximate interface curves, under the rather mild mesh condition $\Delta t / \Delta x \leqslant$ constant.

These results are an extension of our previous work [5] in which, together with E. DiBenedetto, we presented a complete analysis of an explicit finite-difference scheme for the problem (1.1)-(1.2). Although convenient for purposes of exposition, that scheme was of limited practical significance because of the usual parabolic stability condition for explicit schemes, $\Delta t / \Delta x^{2} \leqslant$ constant. Nevertheless, most of the techniques and arguments used there are applicable here. We shall not, therefore, repeat all the details.

The plan of this paper is as follows. In the remainder of this section we give a detailed description of our algorithm. In Section 2 we derive the basic estimates on the approximate solutions which are needed for the convergence results and error bounds. Now, the feature of our scheme which makes it most attractive computationally is that the required conditions on the mesh parameters are minimal. Since

Received May 5, 1983; revised November 1, 1984

1980 Mathematics Subject Classification. Primary 65M15, 35K65.

1985 American Mathematical Society $0025-5718 / 85 \$ 1.00+\$ .25$ per page 
these conditions are precisely those under which the basic estimates of Section 2 hold, we present these estimates in complete detail. On the other hand, once the approximate solutions and interface curves are known to satisfy these basic estimates, the proof of convergence and the derivation of error bounds are nearly identical to those in the above-mentioned work [5] for the explicit scheme. We therefore present these results in Section 3 with only brief indications of their proofs.

We shall assume throughout that the initial function $v_{0}$ satisfies

$$
\begin{gathered}
0 \leqslant v_{0}(x) \leqslant M, \quad x \in \mathbf{R} ; \\
\left|v_{0}(x)-v_{0}(y)\right| \leqslant L .|x-y|, \quad x, y \in \mathbf{R} ;
\end{gathered}
$$

Under these conditions, it is known that (1.1)-(1.2) has a unique solution $v$ satisfying $0 \leqslant v(x) \leqslant M$; $v$ is Lipschitz continuous in $x$ and Hölder continuous in $t$ with exponent $1 / 2$; and spt $v(\cdot, t)=\left[\zeta_{l}(t), \zeta_{r}(t)\right]$, where the Lipschitz curves $\zeta_{l}$ and $\zeta_{r}$ evolve according to

$$
\begin{gathered}
\dot{\zeta}_{l}(t)=-\frac{m}{m-1} v_{x}\left(\zeta_{l}(t)+0, t\right), \\
\dot{\zeta}_{r}(t)=-\frac{m}{m-1} v_{x}\left(\zeta_{r}(t)-0, t\right) \quad \text { a.e. }
\end{gathered}
$$

Here $v_{x}\left(\zeta_{l}(t)+0, t\right)$ is the limit of $v_{x}(x, t)$ as $x \rightarrow \zeta_{l}(t)$ from the right; similarly, for $v_{x}\left(\zeta_{r}(t)-0, t\right)$. See [1]-[4], [9]. See also [6] and [8] for related work on the numerical solution of (1.1)-(1.2).

We now give a detailed description of our algorithm. Let $\Delta t$ and $\Delta x$ be increments in $t$ and $x$, and let $t_{n}=n \Delta t$ and $x_{k}=k \Delta x$ for integers $n$ and $k$. The approximations to $v\left(x_{k}, t_{n}\right), \zeta_{l}\left(t_{n}\right)$, and $\zeta_{r}\left(t_{n}\right)$ will be denoted by $v_{k}^{n}, \zeta_{l}^{n}$, and $\zeta_{r}^{n}$, respectively. To start the scheme we set $v_{k}^{0}=v_{0}\left(x_{k}\right), \zeta_{l}^{0}=\zeta_{l}(0)$, and $\zeta_{r}^{0}=\zeta_{r}(0)$. Now given $v_{k}^{n}, \zeta_{l}^{n}$, and $\zeta_{r}^{n}$, we proceed as follows. Define

$$
K_{r}^{n}=\max \left\{k: x_{k+1} \leqslant \zeta_{r}^{n}\right\}
$$

and

$$
s_{r}^{n}=\zeta_{r}^{n}-x_{K_{r}^{n}} .
$$

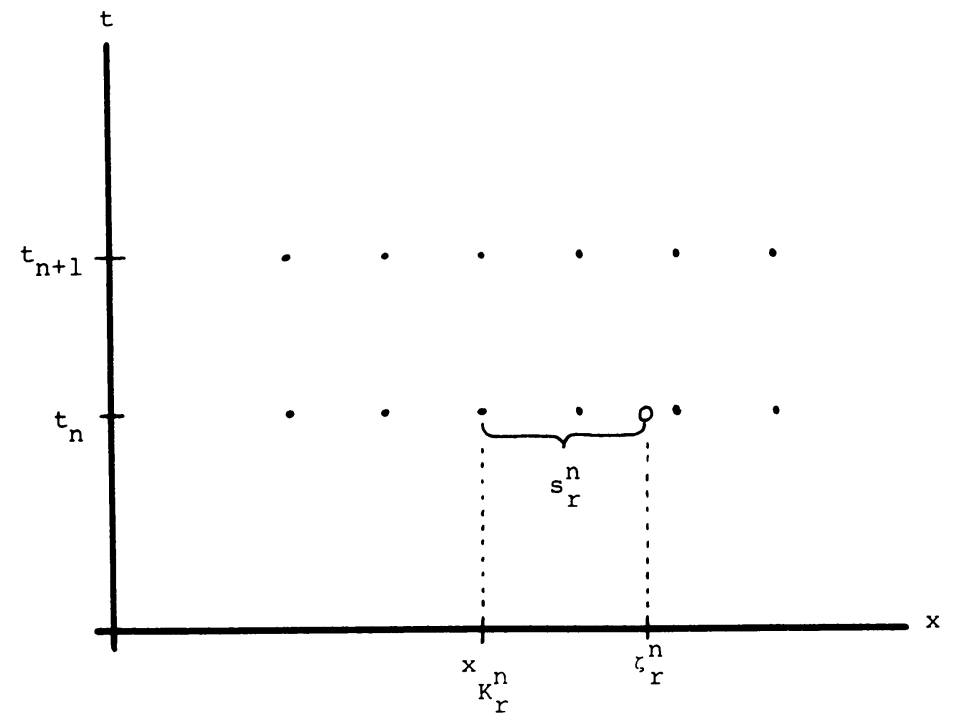


Then in analogy with (1.3), we compute $\zeta_{r}^{n+1}$ from

$$
\zeta_{r}^{n+1}=\zeta_{r}^{n}+\frac{m \Delta t}{m-1} \frac{v_{K_{r}^{n}}^{n}}{s_{r}^{n}}
$$

$K_{l}^{n}, s_{l}^{n}$, and $\zeta_{l}^{n}$ are defined in a similar way. Then for $K_{l}^{n} \leqslant k \leqslant K_{r}^{n}$, we compute $v_{k}^{n+1}$ from the implicit finite-difference equation

$$
\frac{v_{k}^{n+1}-v_{k}^{n}}{\Delta t}=m v_{k}^{n} \frac{A v_{k}^{n+1}}{\Delta x^{2}}+\varepsilon \frac{A v_{k}^{n}}{\Delta x^{2}}+\frac{m}{m-1}\left(\frac{v_{k+1}^{n}-v_{k-1}^{n}}{2 \Delta x}\right)^{2},
$$

where $A$ is the difference operator

$$
(A v)_{k}=v_{k+1}-2 v_{k}+v_{k-1},
$$

and $\varepsilon$ is an $O(\Delta x)$ viscosity parameter to be defined below. For $x_{K_{r}^{n}}<x_{k} \leqslant \zeta_{r}^{n+1}$, we compute $v_{k}^{n+1}$ from the linear interpolation

$$
v_{k}^{n+1}=v_{K_{r}^{n}}^{n+1} \frac{\zeta_{r}^{n+1}-x_{k}}{\zeta_{r}^{n+1}-x_{K_{r}^{n}}}
$$

and similarly at the left-hand interface. And finally we set $v_{k}^{n+1}=0$ for $x_{k} \notin$ $\left[\zeta_{l}^{n+1}, \zeta_{r}^{n+1}\right]$.

Setting $\beta=\Delta t / \Delta x^{2}$, we can rewrite (1.7) as

$$
\begin{aligned}
v_{k}^{n+1}= & v_{k}^{n}+m \beta v_{k}^{n} A v_{k}^{n+1}+\beta \varepsilon A v_{k}^{n} \\
& +\frac{m \Delta t}{m-1}\left(\frac{v_{k+1}^{n}-v_{k-1}^{n}}{2 \Delta x}\right)^{2}, \quad K_{l}^{n} \leqslant k \leqslant K_{r}^{n} .
\end{aligned}
$$

Clearly, the computation of $v_{K_{i}^{n}}^{n+1}, \ldots, v_{K_{r}^{n}}^{n+1}$ requires only the solution of linear, tridiagonal systems of equations. As we shall see in Section 2, this system is uniquely solvable; and, owing to the presence of $\varepsilon$, the bounds [A1] and [A2] persist for the computed, approximate solution. In addition, we shall establish the second-derivative estimate

$$
A v_{k}^{n} / \Delta x^{2} \geqslant- \text { const } / t_{n} .
$$

This lower bound will prove to be crucial for the analysis of the convergence of the approximate interface curves.

We shall need to assume that the mesh-parameters satisfy the following conditions:

$$
\begin{aligned}
& (6 m /(m-1)+3 m) L \Delta x \leqslant \varepsilon \leqslant \text { const } \Delta x, \\
& \beta[\varepsilon+\max (m, m /(m-1)) L \Delta x] \leqslant 1 / 2 .
\end{aligned}
$$

Observe that, since $\varepsilon=O(\Delta x)$, [A5] imposes an upper bound only on $\Delta t / \Delta x$. We have thus avoided the parabolic stability condition for explicit schemes, $\Delta t / \Delta x^{2} \leqslant$ constant.

2. Basic Estimates. Throughout this section we assume that the initial function $v_{0}$ satisfies [A1]-[A3], and that the mesh parameters satisfy [A4] and [A5].

LEMMA 2.1. The scheme (1.9) is uniquely solvable, and the computed solutions $v_{k}^{n}$ satisfy

$$
0 \leqslant v_{k}^{n} \leqslant M
$$


and

$$
\left|\left(v_{k}^{n}-v_{k-1}^{n}\right) / \Delta x\right| \leqslant L
$$

for all $n$ and $k$.

Proof. (2.1) and (2.2) hold for $n=0$ by hypothesis. We assume that they hold at time $t_{n}$ and show that $v_{k}^{n+1} \leqslant M$ for all $k$. The proof that $v_{k}^{n+1} \geqslant 0$ is similar; and the unique solvability then follows by taking $M=0$ when Eqs. (1.9) are homogeneous.

Thus let $\left\{v_{k}^{n+1}\right\}_{k=K_{l}^{n}-1}^{K_{r}^{n}+1}$ solve (1.9), and choose $k$ so that $v_{k}^{n+1}$ is maximal. Because of the linear interpolation near the interface, we may assume that $K_{l}^{n} \leqslant k \leqslant K_{r}^{n}$, so that the difference equation holds at $x_{k}$. We rewrite the last term in (1.9) as

$$
\frac{m \beta \Delta x}{4(m-1)}\left(w_{k+1}^{n}+w_{k}^{n}\right)\left(v_{k+1}^{n}-v_{k-1}^{n}\right)
$$

where $w_{k}^{n}$ is the backward-space difference

$$
w_{k}^{n}=\left(v_{k}^{n}-v_{k-1}^{n}\right) / \Delta x
$$

(1.9) then becomes

$$
\begin{aligned}
\left(1+2 m \beta v_{k}^{n}\right) v_{k}^{n+1}= & m \beta v_{k}^{n}\left(v_{k+1}^{n+1}+v_{k-1}^{n+1}\right)+(1-2 \beta \varepsilon) v_{k}^{n} \\
& +\beta\left[\varepsilon+\frac{m \Delta x}{4(m-1)}\left(w_{k+1}^{n}+w_{k}^{n}\right)\right] v_{k+1}^{n} \\
& +\beta\left[\varepsilon-\frac{m \Delta x}{4(m-1)}\left(w_{k+1}^{n}+w_{k}^{n}\right)\right] v_{k-1}^{n} .
\end{aligned}
$$

Using the fact that $m \beta v_{k}^{n} \geqslant 0$ and the maximality of $v_{k}^{n+1}$, we obtain

$$
\begin{aligned}
v_{k}^{n+1} \leqslant & (1-2 \beta \varepsilon) v_{k}^{n}+\beta\left[\varepsilon+\frac{m \Delta x}{4(m-1)}\left(w_{k+1}^{n}+w_{k}^{n}\right)\right] v_{k+1}^{n} \\
& +\beta\left[\varepsilon-\frac{m \Delta x}{4(m-1)}\left(w_{k+1}^{n}+w_{k}^{n}\right)\right] v_{k-1}^{n} .
\end{aligned}
$$

The induction hypothesis $\left|w_{j}^{n}\right| \leqslant L$ and the mesh conditions [A4] and [A5] now show that the right side of (2.3) is a convex combination of $v_{k-1}^{n}, v_{k}^{n}$, and $v_{k+1}^{n}$, and so is bounded above by $M$. Thus for all $j, v_{j}^{n+1} \leqslant v_{k}^{n+1} \leqslant M$.

Next, we establish the bound (2.2) at time $t_{n+1}$. Subtract from (1.9) the corresponding equation at $x_{k-1}$ and divide by $\Delta x$. The result is that

$$
\begin{aligned}
w_{k}^{n+1}= & w_{k}^{n}+m \beta\left(\frac{v_{k}^{n}+v_{k-1}^{n}}{2}\right) A w_{k}^{n+1} \\
& +m \beta\left(\frac{v_{k}^{n}-v_{k-1}^{n}}{2}\right)\left(w_{k+1}^{n+1}-w_{k-1}^{n+1}\right)+\beta \varepsilon A w_{k}^{n} \\
& +\frac{m \Delta t}{m-1}\left(\frac{w_{k+1}^{n}+2 w_{k}^{n}+w_{k-1}^{n}}{2}\right)\left(\frac{w_{k+1}^{n}-w_{k-1}^{n}}{2 \Delta x}\right), \quad K_{l}^{n}<k \leqslant K_{r}^{n} .
\end{aligned}
$$


Rearranging, we find that

$$
\begin{aligned}
{[1+2 m \beta(} & \left.\left.\frac{v_{k}^{n}+v_{k-1}^{n}}{2}\right)\right] w_{k}^{n+1} \\
= & m \beta v_{k}^{n} w_{k+1}^{n+1}+m \beta v_{k-1}^{n} w_{k-1}^{n+1}+(1-2 \beta \varepsilon) w_{k}^{n} \\
& +\beta\left[\varepsilon+\frac{m \Delta x}{m-1}\left(\frac{w_{k+1}^{n}+2 w_{k}^{n}+w_{k-1}^{n}}{4}\right)\right] w_{k+1}^{n} \\
& +\beta\left[\varepsilon-\frac{m \Delta x}{m-1}\left(\frac{w_{k+1}^{n}+2 w_{k}^{n}+w_{k-1}^{n}}{4}\right)\right] w_{k-1}^{n} .
\end{aligned}
$$

Now, the coefficients of $w_{k+1}^{n+1}$ and $w_{k-1}^{n+1}$ are nonnegative by the induction hypothesis. Therefore, if we choose $k$ so that $w_{k}^{n+1}$ is maximal, and if we assume for the moment that $K_{l}^{n}<k \leqslant K_{r}^{n}$, it then follows that

$$
\begin{aligned}
w_{k}^{n+1} \leqslant & (1-2 \beta \varepsilon) w_{k}^{n}+\beta\left[\varepsilon+\frac{m \Delta x}{m-1}\left(\frac{w_{k+1}^{n}+2 w_{k}^{n}+w_{k-1}^{n}}{4}\right)\right] w_{k+1}^{n} \\
& +\beta\left[\varepsilon-\frac{m \Delta x}{m-1}\left(\frac{w_{k+1}^{n}+2 w_{k}^{n}+w_{k-1}^{n}}{4}\right)\right] w_{k-1}^{n} .
\end{aligned}
$$

Again, the induction hypotheses and the mesh conditions [A4] and [A5] show that the right side of (2.6) is a convex combination of numbers which are less than or equal to $L$. Thus for all $j, w_{j}^{n+1} \leqslant w_{k}^{n+1} \leqslant L$. The proof that $w_{j}^{n+1} \geqslant-L$ is similar.

To complete the proof, we must show that $\left|w_{k}^{n+1}\right| \leqslant L$ when $x_{k}$ is near an interface $\zeta_{l}^{\prime \prime}$ or $\zeta_{r}^{n}$. We shall deal only with the right-hand interface, and for this it will be sufficient to bound $w_{K+1}^{n+1}$, where $K=K_{r}^{n}$. From the definition of $v_{j}^{n+1}$ for $j>K$, (1.8), and from the difference equation (1.9), we have that

$$
\begin{aligned}
w_{K+1}^{n+1}=\frac{0-v_{K}^{n+1}}{s}=-\frac{1}{s}\left[v_{K}^{n}\right. & +m \beta \Delta x v_{K}^{n}\left(w_{K+1}^{n+1}-w_{K}^{n+1}\right) \\
& \left.+\beta \varepsilon \Delta x\left(w_{K+1}^{n}-w_{K}^{n}\right)+\frac{m \Delta t}{m-1}\left(\frac{w_{K+1}^{n}+w_{K}^{n}}{2}\right)^{2}\right],
\end{aligned}
$$

where $s=\zeta_{r}^{n+1}-x_{K_{r}^{n}}$. Using the fact that

$$
w_{K+1}^{n}=-v_{K}^{n} / s_{r}^{n}
$$

and rearranging, we obtain

$$
\begin{aligned}
w_{K+1}^{n+1}= & \frac{1}{s}\left[s_{r}^{n} w_{K+1}^{n}-m \beta \Delta x v_{K}^{n}\left(w_{K+1}^{n+1}-w_{K}^{n+1}\right)-\beta \varepsilon \Delta x\left(w_{K+1}^{n}-w_{K}^{n}\right)\right. \\
& \left.-\frac{m \Delta t}{m-1}\left\{\left(w_{K+1}^{n}\right)^{2}-\left(w_{K+1}^{n}-w_{K}^{n}\right)\left(\frac{3 w_{K+1}^{n}+w_{K}^{n}}{4}\right)\right\}\right] \\
= & w_{K+1}^{n}\left(\frac{s_{r}^{n}-m \Delta t(m-1)^{-1} w_{K+1}^{n}}{s}\right)-\frac{m \beta \Delta x}{s} v_{K}^{n}\left(w_{K+1}^{n+1}-w_{K}^{n+1}\right) \\
& -\frac{1}{s}\left[\beta \varepsilon \Delta x-\frac{m \Delta t}{m-1}\left(\frac{3 w_{K+1}^{n}+w_{K}^{n}}{4}\right)\right]\left(w_{K+1}^{n}-w_{K}^{n}\right) .
\end{aligned}
$$

However, the interface condition (1.6) shows that

$$
s=\zeta_{r}^{n+1}-x_{K_{r}^{n}}=\zeta_{r}^{n}-\frac{m \Delta t}{m-1} w_{K+1}^{n}-x_{K_{r}^{n}}=s_{r}^{n}-\frac{m \Delta t}{m-1} w_{K+1}^{n} .
$$


We therefore obtain from (2.7) that

$$
w_{K+1}^{n+1}=(1-a) w_{K+1}^{n}+a w_{K}^{n}-b\left(w_{K+1}^{n+1}-w_{K}^{n+1}\right),
$$

where

$$
a=\frac{\Delta t}{\Delta x\left(\zeta_{r}^{n+1}-x_{K_{r}^{n}}\right)}\left[\varepsilon-\frac{m \Delta x}{m-1}\left(\frac{3 w_{K_{r}^{n}+1}^{n}+w_{K_{r}^{n}}^{n}}{4}\right)\right]
$$

and

$$
b=\frac{m \Delta t}{\Delta x\left(\zeta_{r}^{n+1}-x_{K_{r}^{n}}\right)} v_{K_{r}^{n}}^{n}
$$

Now, $w_{K+1}^{n+1}=-v_{K}^{n+1} / s \leqslant 0$. Therefore, if $\left|w_{K+1}^{n+1}\right|$ is maximal, $w_{K+1}^{n+1}$ must be negative and minimal, so that $w_{K+1}^{n+1}-w_{K}^{n+1} \leqslant 0$. We therefore obtain from (2.8) that

$$
w_{K+1}^{n+1} \geqslant(1-a) w_{K+1}^{n}+a w_{K}^{n} .
$$

But $a$ is nonnegative by the induction hypothesis and [A4], and

$$
a \leqslant \beta\left(\varepsilon+\frac{m}{m-1} L \Delta x\right) \leqslant 1
$$

by [A5]. We may therefore conclude from (2.11) that $w_{K+1}^{n+1} \geqslant-L$.

In the next lemma we establish a one-sided bound for the discrete second-space derivative of the computed solutions.

LEMMA 2.2. There is a constant $C$ depending only on $M$ and $L$ such that the bound

$$
\frac{A v_{k}^{n}}{\Delta x^{2}}=\frac{v_{k+1}^{n}-2 v_{k}^{n}+v_{k-1}^{n}}{\Delta x^{2}} \geqslant-\frac{C}{t_{n}}
$$

holds for all $k$ and all $n>0$.

Proof. Let $z_{k}^{n}$ be the variable in question. That is,

$$
z_{k}^{n}=\frac{A v_{k}^{n}}{\Delta x^{2}}=\frac{w_{k+1}^{n}-w_{k}^{n}}{\Delta x},
$$

where, as before, $w_{k}^{n}=\left(v_{k}^{n}-v_{k-1}^{n}\right) / \Delta x$. We let $n_{0}$ denote a positive integer, to be chosen later, which depends only on $M$ and $L$. Then for $n \leqslant n_{0}$,

$$
z_{k}^{n} \geqslant-\frac{2 L}{\Delta x}=-\frac{2 L}{t_{n}} \frac{n \Delta t}{\Delta x} \geqslant-\frac{C}{t_{n}},
$$

where $C$ depends only on $M$ and $L$. (Observe that the mesh condition [A5] imposes an upper bound on $\Delta t / \Delta x$ in terms of $M$ and $L$.)

Proceeding by induction, we establish the bound (2.13) at time $t_{n+1}$, where $n+1>n_{0}$. Thus, choose $k$ so that $z_{k}^{n+1}$ is minimal and, without loss of generality, nonpositive. First assume that $x_{k}$ is an interior point; that is, $K_{l}^{n}<k<K_{r}^{n}$. In this case Eq. (2.4) holds at both $x_{k}$ and $x_{k+1}$. We rewrite this equation as

$$
\begin{aligned}
w_{k}^{n+1}= & w_{k}^{n}+m \beta \Delta x\left(\frac{v_{k}^{n}+v_{k-1}^{n}}{2}\right)\left(z_{k}^{n+1}-z_{k-1}^{n+1}\right) \\
& +m \beta \Delta x\left(v_{k}^{n}-v_{k-1}^{n}\right)\left(\frac{z_{k}^{n+1}+z_{k-1}^{n+1}}{2}\right)+\beta \varepsilon \Delta x\left(z_{k}^{n}-z_{k-1}^{n}\right) \\
& +\frac{m \Delta t}{m-1}\left(\frac{w_{k+1}^{n}+2 w_{k}^{n}+w_{k-1}^{n}}{4}\right)\left(z_{k}^{n}+z_{k-1}^{n}\right) .
\end{aligned}
$$


Subtract this from the corresponding equation at $x_{k+1}$ and divide by $\Delta x$. The result is that

$$
\begin{aligned}
z_{k}^{n+1}= & z_{k}^{n}+m \beta\left(\frac{v_{k+1}^{n}+2 v_{k}^{n}+v_{k-1}^{n}}{4}\right) A z_{k}^{n+1} \\
& +m \beta\left(\frac{v_{k+1}^{n}-v_{k-1}^{n}}{2}\right)\left(z_{k+1}^{n+1}-z_{k-1}^{n+1}\right) \\
& +m \Delta t z_{k}^{n}\left(\frac{z_{k+1}^{n+1}+2 z_{k}^{n+1}+z_{k-1}^{n+1}}{4}\right)+\beta \varepsilon A z_{k}^{n} \\
& +\frac{m}{m-1} \frac{\Delta t}{\Delta x}\left(\frac{w_{k+2}^{n}+3 w_{k+1}^{n}+3 w_{k}^{n}+w_{k-1}^{n}}{8}\right)\left(z_{k+1}^{n}-z_{k-1}^{n}\right) \\
& +\frac{m}{m-1} \frac{\Delta t}{8}\left(z_{k+1}^{n}+2 z_{k}^{n}+z_{k-1}^{n}\right)^{2} .
\end{aligned}
$$

Combining terms, we obtain

$$
\begin{aligned}
{[1+} & \left.m \beta\left(\frac{v_{k+1}^{n}+2 v_{k}^{n}+v_{k-1}^{n}}{2}\right)-\frac{m \Delta t}{2} z_{k}^{n}\right] z_{k}^{n+1} \\
= & m \beta\left(\frac{3 v_{k+1}^{n}+2 v_{k}^{n}-v_{k-1}^{n}}{4}+\frac{\Delta x^{2}}{4} z_{k}^{n}\right) z_{k+1}^{n+1} \\
& +m \beta\left(\frac{-v_{k+1}^{n}+2 v_{k}^{n}+3 v_{k-1}^{n}}{4}+\frac{\Delta x^{2}}{4} z_{k}^{n}\right) z_{k-1}^{n+1}+(1-2 \beta \varepsilon) z_{k}^{n} \\
& +\beta\left(\varepsilon+\frac{m}{m-1} \Delta x \bar{w}\right) z_{k+1}^{n}+\beta\left(\varepsilon-\frac{m}{m-1} \Delta x \bar{w}\right) z_{k-1}^{n}+\frac{m}{m-1} \frac{\Delta t}{8} \bar{z}^{2},
\end{aligned}
$$

where $\bar{w}$ and $\bar{z}$ are the obvious averages. Now, since

$$
\Delta x^{2} z_{k}^{n}=v_{k+1}^{n}+2 v_{k}^{n}+v_{k-1}^{n},
$$

the coefficients of $z_{k+1}^{n+1}$ and $z_{k-1}^{n+1}$ in the above equation are nonnegative. And since $z_{k}^{n+1}$ is minimal, we obtain

$$
\begin{aligned}
z_{k}^{n+1} \geqslant & m \Delta t z_{k}^{n} z_{k}^{n+1}+(1-2 \beta \varepsilon) z_{k}^{n}+\beta\left(\varepsilon+\frac{m}{m-1} \Delta x \bar{w}\right) z_{k+1}^{n} \\
& +\beta\left(\varepsilon-\frac{m}{m-1} \Delta x \bar{w}\right) z_{k-1}^{n} .
\end{aligned}
$$

The mesh condition [A4] now shows that the right-hand side here is an increasing function of $z_{k \pm 1}^{n}$. The same is true for the variable $z_{k}^{n}$, if we fix $z_{k}^{n+1} \geqslant-2 L / \Delta x$, since then

$$
m \Delta t z_{k}^{n+1}+1-2 \beta \varepsilon \geqslant 1-2 \beta(\varepsilon+m L \Delta x),
$$

which is nonnegative by [A5]. We therefore have from (2.15) and the induction hypothesis that

$$
z_{k}^{n+1} \geqslant-\left(C / t_{n}\right)\left(1+m \Delta t z_{k}^{n+1}\right) .
$$

And this shows that $z_{k}^{n+1} \geqslant-C / t_{n+1}$ provided that we take $C \geqslant 1 / m$.

To complete the proof, we need to deal with the case that the minimal $z_{k}^{n+1}$ occurs near the interface. Now, when $k \notin\left[K_{l}^{n}, K_{r}^{n}\right], z_{k}^{n+1}$ will be nonnegative because of the linear interpolation used to construct $v$ near the interface, We therefore set $K=K_{r}^{n}$ and assume that $z_{K}^{n+1}$ is minimal. The argument at the left interface is similar. 
First we rewrite (2.8) in the form

$$
w_{K+1}^{n+1}=w_{K+1}^{n}-a \Delta x z_{K}^{n}-b \Delta x z_{K}^{n+1},
$$

where $a$ and $b$ are as defined in (2.9) and (2.10). Next, we have from (2.14) that

$$
\begin{aligned}
w_{K}^{n+1}= & w_{K}^{n}+(c+d) \Delta x z_{K}^{n+1}-(c-d) \Delta x z_{K-1}^{n+1} \\
& +(f+g) \Delta x z_{K}^{n}-(f-g) \Delta x z_{K-1}^{n},
\end{aligned}
$$

where

$$
\begin{gathered}
c=m \beta \frac{v_{K}^{n}+v_{K-1}^{n}}{2}, \quad d=\frac{m \beta \Delta x w_{K}^{n}}{2}, \quad f=\beta \varepsilon, \\
g=\frac{m}{m-1} \frac{\Delta t}{\Delta x} \frac{w_{k+1}^{n}+2 w_{k}^{n}+w_{k-1}^{n}}{4} .
\end{gathered}
$$

Subtracting (2.17) from (2.16) and dividing by $\Delta x$, we obtain

$$
z_{K}^{n+1}=(1-a-f-g) z_{K}^{n}+(f-g) z_{K-1}^{n}-(b+c+d) z_{K}^{n+1}+(c-d) z_{K-1}^{n+1} .
$$

Now,

$$
c-d=m \beta v_{K}^{n} \geqslant 0,
$$

so that, since $z_{K}^{n+1}$ is minimal,

$$
(1+b+2 d) z_{K}^{n+1} \geqslant(1-a-f-g) z_{K}^{n}+(f-g) z_{K-1}^{n} .
$$

We have that $f-g \geqslant 0$ by [A4]. And from (2.12) and (2.17),

$$
\begin{aligned}
1-a-f-g & \geqslant 1-\left(\beta \varepsilon+\frac{m}{m-1} L \frac{\Delta t}{\Delta x}\right)-\beta \varepsilon-\frac{m}{m-1} \frac{\Delta t}{\Delta x} L \\
& =1-2 \beta\left(\varepsilon+\frac{m}{m-1} L \Delta x\right),
\end{aligned}
$$

which is nonnegative by [A5]. Our induction hypothesis applied to (2.19) then shows that

$$
(1+b+2 d) z_{K}^{n+1} \geqslant(1-a-2 g)\left(-C / t_{n}\right) .
$$

We need to estimate the coefficients in this inequality. First, we have from the construction of $s_{r}^{n}$ (see (1.5)) that

$$
\begin{aligned}
\zeta_{r}^{n+1}-x_{K_{r}^{n}} & =s_{r}^{n}+\zeta_{r}^{n+1}-\zeta_{r}^{n} \leqslant 2 \Delta x+\frac{m}{m-1} L \Delta t \\
& \leqslant 2 \Delta x+\varepsilon \frac{\Delta t}{\Delta x}=\Delta x(2+\varepsilon \beta) \leqslant \frac{5}{2} \Delta x
\end{aligned}
$$

by $[\mathrm{A} 4]$ and $[\mathrm{A} 5]$. Thus

$$
\beta^{\prime} \equiv \Delta t / \Delta x\left(\zeta_{r}^{n+1}-x_{K_{r}^{n}}\right) \geqslant 2 \beta / 5,
$$

so that, from (2.9) and (2.18),

$$
\begin{aligned}
1-a-2 g & \leqslant 1-\beta^{\prime}\left(\varepsilon-\frac{m}{m-1} L \Delta x\right)+\frac{2 m}{m-1} L \frac{\Delta t}{\Delta x} \\
& \leqslant 1-\frac{2}{5} \beta\left(\varepsilon-\frac{6 m}{m-1} L \Delta x\right) \leqslant 1-\frac{6}{5} m \beta L \Delta x
\end{aligned}
$$

by [A4]. Observe also that $1-a-2 g$ is nonnegative, because, as we showed above, both $1-a-f-g$ and $f-g$ are nonnegative. Finally,

$$
1+b+2 d \geqslant 1+2 d \geqslant 1-m \beta L \Delta x>0
$$


by [A5]. Substituting the estimates (2.21) and (2.22) into (2.20), we therefore obtain

$$
z_{K}^{n+1} \geqslant \frac{1-\frac{6}{5} m \beta L \Delta x}{1-m \beta L \Delta x}\left(-\frac{C}{t_{n}}\right) \geqslant(1-\delta)\left(-\frac{C}{t_{n}}\right)
$$

where $\delta$ depends only on $M$ and $L$. And this shows that

$$
z_{K}^{n+1} \geqslant-C / t_{n+1}
$$

provided that $n \geqslant n_{0}$, where $n_{0}$ depends only on $M$ and $L$.

The following estimates are consequences of Lemmas 2.1 and 2.2.

LEMMA 2.3. Given $T>0$, there is a constant $C$ depending only on $M, L$, and $T$ such that the bounds

$$
\sum_{k}\left|\frac{A v_{k}^{n}}{\Delta x^{2}}\right| \Delta x, \sum_{k}\left|\frac{v_{k}^{n+1}-v_{k}^{n}}{\Delta t}\right| \Delta x \leqslant C\left(1+\frac{1}{t_{n}}\right)
$$

hold for $t_{n} \leqslant T$.

LEMMA 2.4. Given $T>0$, there is a constant $C$ depending only on $M, L$, and $T$ such that the inequality

$$
\left|v_{k}^{n}-v_{k}^{m}\right| \leqslant c\left|t_{n}-t_{m}\right|^{1 / 2}
$$

holds for $t_{n}$ and $t_{m}$ in $[0, T]$ and for all $k$.

Lemma 2.3 follows directly from the bound (2.13) and the difference equation (1.7). And Lemma 2.4 can be proved by employing the discrete version of a technique due to Kružkov [7] for deducing a modulus of continuity in time from a known modulus of continuity in space for solutions of certain parabolic equations. The proofs of Lemmas 2.3 and 2.4 are nearly identical to those of Lemmas 2.6 and 2.7 in [5], and in any case they impose no further constraints upon the mesh parameters. We therefore omit these proofs.

3. Convergence and Error Bounds. We let $h=(\Delta t, \Delta x)$ be a point in $\mathbf{R}_{+}^{2}$ whose coordinates satisfy the mesh conditions [A4]-[A5]. And we define approximate solutions $v^{h}$ and approximate interface curves $\zeta_{r, l}^{h}$ by prolonging the sequences $\left\{v_{k}^{n}\right\}$ and $\left\{\zeta_{r, l}^{n}\right\}$ by piecewise-linear interpolation. For example, for $t_{n-1} \leqslant t \leqslant t_{n}$,

$$
\zeta_{r}^{h}(t)=\zeta_{r}^{n-1}+\frac{\zeta_{r}^{n}-\zeta_{r}^{n-1}}{\Delta t}\left(t-t_{n-1}\right)
$$

And for $(x, t)$ in the triangle with vertices $\left(x_{k}, t_{n-1}\right),\left(x_{k}, t_{n}\right)$, and $\left(x_{k+1}, t_{n}\right)$,

$$
v^{h}(x, t)=v_{k}^{n}+\frac{v_{k+1}^{n}-v_{k}^{n}}{\Delta x}\left(x-x_{k}\right)+\frac{v_{k}^{n}-v_{k}^{n-1}}{\Delta t}\left(t-t_{n}\right),
$$

and so on. Lemmas 2.1-2.4 then show that

$$
\begin{gathered}
0 \leqslant v^{h}(x, t) \leqslant M \\
\left|v_{x}^{h}(x, t)\right| \leqslant L \quad \text { a.e., }
\end{gathered}
$$

and for $t, t_{1}, t_{2} \leqslant T$,

$$
\begin{gathered}
\left|v^{h}\left(x, t_{2}\right)-v^{h}\left(x, t_{1}\right)\right| \leqslant C\left|t_{2}-t_{1}\right|^{1 / 2} \\
\left\|v_{t}^{h}(\cdot, t)\right\|_{1, \mathbf{R}}, \int\left|v_{x x}^{h}(x, t)\right| d x \leqslant C\left(1+\frac{1}{t}\right) .
\end{gathered}
$$


(Of course, $v_{x x}^{h}$ is a measure.) As a consequence of these estimates we have the following convergence result.

THEOREM 3.1. Assume that the initial function $v_{0}$ satisfies [A1]-[A3], and let $v$ be the unique solution of (1.1)-(1.2). Let $v^{h}, \zeta_{r}^{h}$, and $\zeta_{l}^{h}$ be as constructed above, where the mesh parameters are assumed to satisfy [A4]-[A5]. Then given $T>0$,

$$
\begin{gathered}
v^{h} \rightarrow \text { v uniformly in } \mathbf{R} \times[0, T], \\
v_{x}^{h} \rightarrow v_{x} \text { in } L^{p}(\mathbf{R} \times[0, T]) \text { for } p<\infty,
\end{gathered}
$$

and

$$
\zeta_{l}^{h}, \zeta_{r}^{h} \rightarrow \zeta_{l}, \zeta_{r} \text { uniformly in }[0, T]
$$

Proof. The compactness of the nets $\left\{v^{h}\right\}$ and $\left\{v_{x}^{h}\right\}$ is evident from (3.1)-(3.4). That their limit points coincide with $v$ and $v_{x}$ follows from the uniqueness of solutions of (1.1)-(1.2) and the fact that our difference equation (1.7) is consistent with (1.1). The convergence of the approximate interface curves is more subtle, requiring a careful coupling of the one-sided bound for $v_{x x}^{h}$ in Lemma 2.2 with the interface condition (1.3) and its discretization (1.6). Complete details may be found in Section 3 of [5].

Finally, we can prove the following error bounds for the approximate solutions and interface curves.

THEOREM 3.2. Assume that the hypotheses of Theorem 3.1 are in force. Then given $T>0$ there is a constant $C$ such that

$$
\begin{gathered}
\left\|v^{h}-v\right\|_{p, \mathbf{R} \times[0, T]} \leqslant C H^{1 / p}, \\
\left\|v^{h}(\cdot, t)-v(\cdot, t)\right\|_{\infty, \mathbf{R}} \leqslant C \min \left[(H / t)^{1 / p+3}, t^{1 / 2}+\Delta x\right], \\
\left\|\zeta_{r, l}^{h}-\zeta_{r, l}\right\|_{\infty,[0, T]} \leqslant C H^{1 / 2(p+3)} .
\end{gathered}
$$

Here

$$
p= \begin{cases}(m+1) /(m-1), & 1<m<2 \\ m+1, & 2 \leqslant m\end{cases}
$$

and

$$
H=\left(\Delta x^{\alpha}+\Delta t^{\alpha / 2}\right)|\log \Delta x|
$$

where

$$
\alpha= \begin{cases}1, & 1<m<2, \\ 1 /(m-1), & 2 \leqslant m .\end{cases}
$$

Proof. To prove (3.5) we exploit the built-in stability of the solution operator for (1.1)-(1.2). Thus $v^{h}-v$ can be estimated in terms of the weak truncation error-the extent to which $v^{h}$ fails to be an exact weak solution of (1.1)-(1.2). (3.6) then follows by applying an elementary interpolation inequality to the estimate (3.5) and using the regularity results of Section 2. And (3.7) is proved by exploiting the one-sided bound for $v_{x x}^{h}$ of Lemma 2.2 and the interface conditions (1.3) and (1.6) to couple the time evolution of the $L^{\infty}$-error in $v^{h}$ to that in $\zeta^{h}$. The details are nearly identical to those in Sections 4 and 5 of [5]. 
We remark that if the initial function $v_{0}$ satisfies $\partial^{2} v_{0} / \partial x^{2} \geqslant-C$ for some constant $C$, then this bound persists both for $v^{h}(\cdot, t)$ and for $v(\cdot, t)$. In this case the $1 / t_{n}$ may be omitted from the estimate in Lemma 2.2, and, as a result, the $|\log \Delta x|$ term may be omitted from the definition of $H$ in the above error bounds. If, in addition, $v_{0}$ is concave, then so are $v^{h}(\cdot, t)$ and $v(\cdot, t)$. In this case $v_{x x}^{h}$ and $v_{t}^{h}$ become bounded in $L^{\infty}$, and the error bounds (3.5)-(3.7) can be improved somewhat.

Finally, if $\dot{\zeta}$ and $\dot{\zeta}^{h}$ are known a priori to be bounded away from 0 , then the proof shows that the bound for $\left\|\zeta^{h}-\zeta\right\|_{\infty}$ in (3.7) becomes $C H^{1 /(p+3)}$.

Our computational experience indicates that the estimates appearing in Theorem 3.2 significantly overstate the actual errors. This may be due in part to the fact that these bounds were derived under minimal smoothness hypotheses on the exact solutions. On the other hand, we have found that, even in the special case that $v_{0}$ is concave and $v$ has bounded derivatives of all orders on its support, the $L^{p}$ error in $v^{h}$ will be at best $O(\Delta x)$. This is not unexpected, since the artificial viscosity parameter $\varepsilon$ is itself $O(\Delta x)$.

Department of Mathematics

Indiana University

Bloomington, Indiana 47405

1. D. G. Aronson, "Regularity properties of flows through porous media," SIAM J. Appl. Math., v. 17, 1969, pp. 461-467.

2. D. G. ARONSON, "Regularity properties of flows through porous media: the interface," Arch. Rational Mech. Anal., v. 37, 1970, pp. 1-10.

3. D. G. ARONSON, "Regularity properties of flows through porous media: a counterexample," SIAM J. Appl. Math., v. 19, 1970, pp. 200-307.

4. D. G. ARONSON \& Ph. Benilan, "Régularité des solutions de l'équation des milieux poreus dans $\mathbf{R}^{N}, "$ C. R. Acad. Sci. Paris Vie Académique, v. 288, 1979, pp. 103-105.

5. E. DiBenedetto \& David HofF, "An interface tracking algorithm for the porous medium equation,” Trans. Amer. Math. Soc., v. 288, 1984, pp. 463-500.

6. J. L. GRaveleau \& P. Jamet, "A finite difference approach to some degenerate nonlinear parabolic equations,” SIAM J. Appl. Math., v. 20, 1971, 199-223.

7. S. N. KRUŽKov, "Results concerning the nature of the continuity of solutions of parabolic equations and some of their applications," Math. Notes, v. 6, no. 1, 1969, pp. 517-523.

8. M. Mimura \& K. Tomoeda, “Numerical approximations for interface curves to a porous media equation." (To appear.)

9. O. A. Oleĭnik, A. S. Kalashnikov \& Chzou Yui-Lin, "The Cauchy problem and boundary problems for equations of the type of unsteady filtration," Izv. Akad. Nauk SSSR Ser. Mat., v. 22, 1958, pp. 667-704. (Russian)

10. M. Muskat, The Flow of Homogeneous Fluids Through Porous Media, McGraw-Hill, New York, 1937. 